\title{
Somatostatin Receptor Expression in GH-Secreting Pituitary Adenomas Treated with Long-Acting Somatostatin Analogues in Combination with Pegvisomant
}

\author{
Sanne E. Franck ${ }^{a} \quad$ Federico Gatto $^{a} \quad$ Aart Jan van der Lely ${ }^{a}$ \\ Joseph A.M.J.L. Janssen ${ }^{a} \quad$ Alof H.G. Dallenga ${ }^{b} \quad$ A. Paul Nagtegaalc \\ Leo J. Hofland ${ }^{a}$ Sebastian J.C.M.M. Neggers ${ }^{a}$ \\ ${ }^{a}$ Endocrinology Section, Department of Internal Medicine, and Departments of ${ }^{b}$ Neurosurgery and \\ 'Otorhinolaryngology, Pituitary Center Rotterdam, Erasmus University Medical Center Rotterdam, Rotterdam, \\ The Netherlands
}

\section{Key Words}

Acromegaly · Pituitary adenoma - Growth hormone . Insulin-like growth factor I. Somatostatin receptor . Somatostatin analogues · Pegvisomant · Growth hormone receptor antagonist

\begin{abstract}
Background: Growth hormone-secreting pituitary adenomas (somatotroph adenoma) predominantly express somatostatin receptors (SSTRs) subtypes 2 and 5 . Higher SSTR2 expression on somatotroph adenomas results in a better response to somatostatin analogues (SSAs), which preferentially bind, but also downregulate, SSTR2. The effect of the combined treatment with SSAs and the GH receptor antagonist pegvisomant (PEGV) on SSTR expression in somatotroph adenomas is currently unknown. Aim of the Study: To assess SSTR2 and SSTR5 expression in three groups of somatotroph adenomas: drug-naive, treated with long-acting (LA) SSA monotherapy, or LA-SSA/PEGV combination therapy before surgery. Additionally, we evaluated the required PEGV dose
\end{abstract}

\section{KARGER}

E-Mail karger@karger.com www.karger.com/nen
This article is licensed under the Creative Commons AttributionNonCommercial-NoDerivatives 4.0 International License (CC BYNC-ND) (http://www.karger.com/Services/OpenAccessLicense) Usage and distribution for commercial purposes as well as
tribution of modified material requires written permission. to achieve insulin-like growth factor I (IGF-I) normalization in relation to the SSTR expression. Materials and Methods: At our Pituitary Center Rotterdam, we selected acromegalic patients who underwent transsphenoidal neurosurgery. All patients were eventually treated with LA-SSA/PEGV combination therapy during their medical history. SSTR2 and SSTR5 expression in somatotroph adenoma tissues was determined using immunohistochemistry. Results: Out of 39 somatotroph adenoma tissue samples, 23 were drug-naive, 9 received pretreatment with LA-SSA and 7 LA-SSA/PEGV combined treatment. SSTR2 expression was significantly higher in treatment-naive compared to combined treatment somatotroph adenomas $(p=0.048)$, while SSTR5 expression did not differ. Noteworthy, SSTR2 expression in naive somatotroph adenoma tissues was inversely correlated with the required PEGV dose to achieve IGF-I normalization during postsurgical medical treatment $(\rho=-0.538, p=0.024)$. Conclusion: In our specific cohort, the SSTR2 expression was

S.E.F. and F.G. contributed equally and share first authorship.

S.E. Franck, MD

Endocrinology Section, Department of Internal Medicine Erasmus University Medical Center, PO Box 2040

NL-3000 CA Rotterdam (The Netherlands)

E-Mail s.franck@erasmusmc.nl 
lower in patients pretreated with LA-SSA/PEGV compared to the drug-naive acromegalic patients. Additionally, the SSTR2 expression in treatment-naive somatotroph adenoma tissues was inversely correlated with the required PEGV dose to achieve IGF-I normalization.

(C) 2016 The Author(s)

Published by S. Karger AG, Basel

\section{Introduction}

Growth hormone-secreting pituitary adenomas (somatotroph adenomas) express different subtypes of somatostatin receptors (SSTRs), predominantly SSTR subtypes 2 (SSTR2) and 5 (SSTR5) [1], which play a major role in reducing $\mathrm{GH}$ secretion and thereby insulin-like growth factor I (IGF-I) levels. Long-acting somatostatin analogues (LA-SSAs) have a high binding affinity for SSTR2 and a moderate affinity for SSTR5. According to current guidelines, LA-SSAs are considered to be the first-line medical treatment modality after unsuccessful surgery as well as primary treatment option in selected cases $[2,3]$. However, a number of studies published on the efficacy of LA-SSAs in acromegaly show that LA-SSA treatment alone fails to reach complete normalization of IGF-I levels in about $50 \%$ of the cases. A recent metaanalysis reported an average control rate of $56 \%$ and $55 \%$ for GH and IGF-I normalization, respectively, during monotherapy with LA-SSA [4]. However, due to selection bias, this efficacy rate is probably an overestimation. In unselected treatment-naive patients, an LA-SSA efficacy rate of $40 \%$ seems to be more common $[5,6]$. Tumor shrinkage with LA-SSA was observed in $63-75 \%$ of these primary-treated patients [6].

The expression of SSTR2 on somatotroph adenoma cell membrane is significantly and positively correlated with the efficacy of LA-SSAs in suppressing GH and IGF-I levels in vitro, and it is also associated with IGF-I normalization in acromegalic patients [7-11]. In this light, a recent study from our group showed that an SSTR2 immunoreactivity score (IRS) of at least 5 had a sensitivity of $86 \%$ and a specificity of $91 \%$ in predicting IGF-I control during adjuvant LA-SSA treatment [9]. Additionally, SSTR2 expression and tumor volume reduction after LA-SSA treatment are positively correlated [11, 12]. Moreover, tumors that are partial responders and nonresponders to monotherapy with LA-SSAs seem to have lower SSTR2 mRNA expression and higher SSTR5 mRNA expression compared to full responders on LASSAs [13]. An effective treatment option to normalize IGF-I levels in partial-responder patients is the addition

Combination Treatment and SSTR

Expression in Acromegaly of the $\mathrm{GH}$ receptor antagonist pegvisomant (PEGV) to first-line medical treatment with LA-SSA $[14,15]$. To the best of our knowledge, the expression of SSTR2 and SSTR5 on somatotroph adenoma tissues from acromegalic patients treated with PEGV (together with LA-SSAs) before surgery is currently unknown.

Based on the finding that the response to LA-SSA treatment is mainly driven by the expression of SSTR2, a reduced expression of SSTR2 in untreated somatotroph adenomas at baseline, probably necessitates combined treatment because of (partial) resistance to LA-SSA treatment. However, we cannot exclude feedback mechanisms like the drop in IGF-I levels during medical treatment and an associated increase in hypothalamic growth hormonereleasing hormone (GHRH) levels, which theoretically also can influence the expression of SSTR 2 and SSTR 5 on the somatotroph adenoma cell membrane. To investigate the effects of the addition of PEGV to LA-SSAs on SSTR expression, we assessed the expression of SSTR subtypes 2 and 5 in three groups of acromegalic patients: drugnaive (naive group), treated with long-acting SSA monotherapy (mono-LA-SSA group) before surgery, and treated with the combination of LA-SSAs and PEGV (combined group) before surgery. Since all patients included in the present study received LA-SSA/PEGV combined treatment during their clinical history, we were able to assess the required PEGV dose (added to LA-SSAs) needed after surgery to achieve the normalization of IGF-I levels. Therefore, we additionally evaluated the required PEGV dose in relation to the expression of SSTR2 and SSTR5, in order to observe indirectly the partial resistance to LASSA. The underlying hypotheses were (1) various medical pretreatment modalities could differently affect the SSTR2 and SSTR5 expression on somatotroph adenomas, and (2) the SSTR expression could affect postsurgical PEGV dosing in combination with LA-SSA.

\section{Methods}

Patient and Somatotroph Adenoma Tissue Selection

Data collection of acromegalic patients was performed at our Rotterdam Pituitary Center, and a retrospective evaluation was carried out. We used a database including acromegalic patients which were all eventually treated with PEGV in combination with LA-SSA $(n=141)$, and we selected those patients who underwent transsphenoidal neurosurgery $(n=66)$, representing a total of 71 transsphenoidal surgeries. Five patients underwent a second surgery; of these patients, we only included the tissue samples of the first surgery. Besides medical history data, a somatotroph adenoma paraffin-embedded tissue sample had to be available in order to perform immunohistochemistry. We selected 39 somatotroph adenoma tis-

Neuroendocrinology 2017;105:44-53

DOI: $10.1159 / 000448429$ 


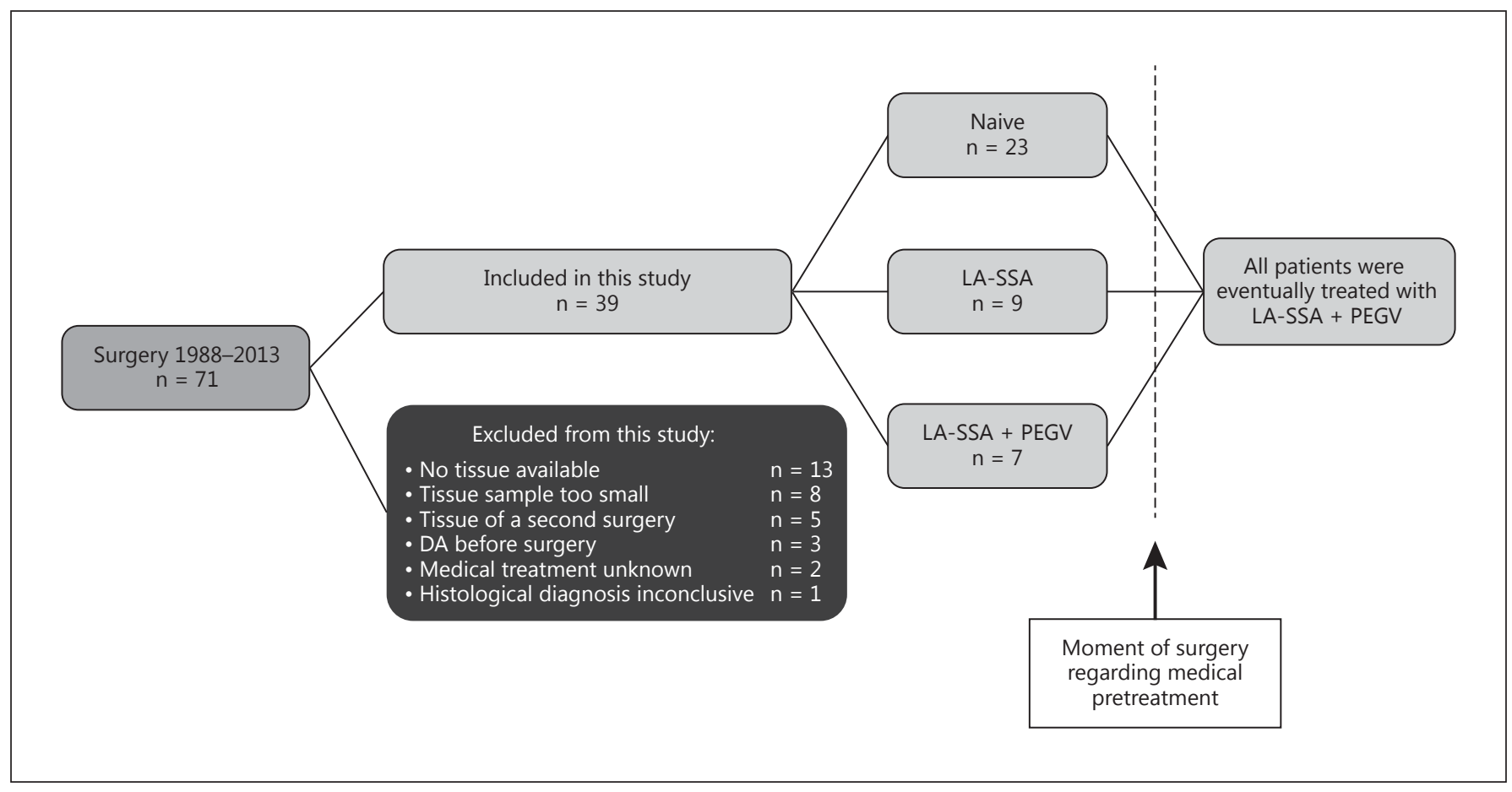

Fig. 1. Flowchart of the selection procedure in this study. Patients who underwent transsphenoidal surgery and eventually were treated with LA-SSA in combination with PEGV, were selected and tissues were collected. The number of tissues (n), which rep- resents the number of patients, as we excluded tissues from a second surgery. DA = Dopamine agonist; Naive = no previous medical treatment; LA-SSA = long-acting somatostatin analogues; PEGV = pegvisomant. sues obtained from 39 patients. A flowchart representing the selection procedure of the somatotroph adenoma tissues for our study is depicted in figure 1. No patient underwent radiotherapy before surgery. Three groups of different medical pretreatment modalities before surgery were identified: drug-naive patients, patients on monotherapy with LA-SSAs and patients treated with LA-SSAs in combination with PEGV. Table 1 shows patient characteristics of the three pretreatment groups before surgery. The mono-LA-SSA group was treated with LA-SSAs for a median of 6 months; the combined group was treated for a median of 20 months with LASSAs and for a median of 13 months with PEGV before surgery. PEGV treatment was added to the highest dose of LA-SSA by weekly injections. For starting doses of PEGV and the protocol of PEGV dose titration to achieve normal IGF-I levels see Neggers et al. [14]. Magnetic resonance imaging was used to assess tumor volume as macro- versus microadenomas at time of diagnosis.

All patients selected for this study were eventually treated with LA-SSA in combination with PEGV after surgery and were subdivided into partial and full responders to monotherapy with LASSAs. Most of the patients included in this study were partial responders to LA-SSAs $(n=31)$ and were considered to have a minimal decrease of $15 \%$ in their GH and IGF-I levels and still have elevated IGF-I serum levels [ $>1.2 \times$ upper limit of normal (ULN)] after at least 6 months on the highest dose of LA-SSAs (Sandostatin LAR $30 \mathrm{mg}$ or lanreotide autogel $120 \mathrm{mg}$ every 28 days). These patients required PEGV in combination with LA-SSA in order to achieve normalized IGF-I levels. Partial responders had a median
IGF-I decrease of $-28.4 \%$ (interquartile range, IQR: -21.2 to -43.4 ) after LA-SSA monotherapy. Full responders to LA-SSAs $(\mathrm{n}=8)$ achieved normal IGF-I levels during monotherapy with LA-SSAs and received PEGV during a clinical trial aimed to investigate aspects of quality of life [16]. Full responders had a median IGF-I decrease of $-61.9 \%$ (IQR: -53.5 to -67.3 ) after LA-SSA monotherapy. In order to subdivide the total cohort into partial and full responders, the response to monotherapy with LA-SSA could have been evaluated before or after surgery. Table 2 provides general, biochemical and somatotroph adenoma characteristics of partial and full responders to the monotherapy with LA-SSAs. When investigating the required PEGV dose to achieve IGF-I normalization in relation to the SSTR2 expression, we included only the partial-responder patients, not pretreated before surgery, in order to achieve a clearer indication from a homogeneous cohort. Permission from the Institutional Review Board of the Erasmus Medical Center Rotterdam was obtained, and all patients gave their written informed consent.

\section{Hormone Assays}

Serum IGF-I levels were measured with a radioimmunoassay [Medgenix Diagnostics, Fleurus, Belgium; intraassay coefficient of variation $(\mathrm{CV}) 6.1 \%$, interassay CV 9.9\%], an immunoradiometric assay (Diagnostic Systems Laboratories, Webster, Tex., USA, intraassay CV 3.9\%, interassay CV 4.2\%) and the Immulite 2000 assay, a solid-phase, enzyme-labelled chemiluminescent immunometric assay (DPC Biermann GmbH/Siemens, Fernwald, Germa- 


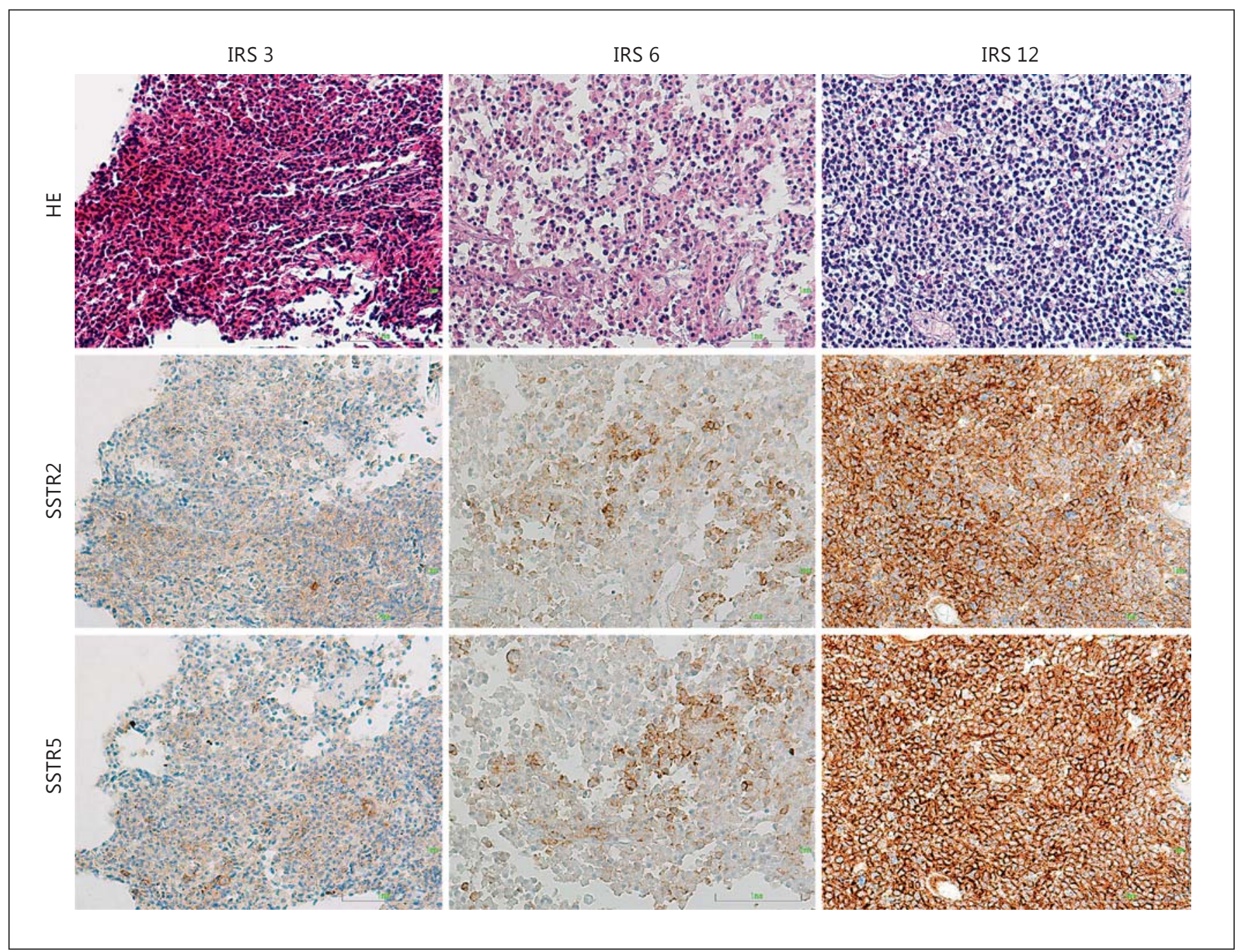

Fig. 2. Heterogeneous expression patterns of SSTR2 and SSTR 5 on somatotroph adenomas scored by the immunoreactivity score (IRS). IRS 3 represents a low, IRS 6 an intermediate and IRS 12 a high SSTR2 and SSTR5 expression pattern. Photography was per- formed during $\times 200$ magnification. $\mathrm{HE}=$ Haematoxylin; SSTR2 $=$ somatostatin receptor subtype 2 ; SSTR 5 = somatostatin receptor subtype 5 . ny; intraassay variability of $2-5 \%$, interassay variability of $3-7 \%$ ). IGF-I age-adjusted reference ranges were used in accordance with an earlier report [17]. IGF-I levels were evaluated just before surgery, during monotherapy of LA-SSA and after surgery when every patient was eventually treated with LA-SSA in combination with PEGV. Over time, IGF-I assays were replaced by one another. We therefore chose to express IGF-I levels only as upper limits of normal (ULN) and not as absolute values.

\section{Immunohistochemistry}

Thirty-nine somatotroph adenoma tissues were available for hematoxylin staining and immunostaining of SSTR2 and SSTR5. Formalin-fixed paraffin-embedded tumor samples were cut into sequential $4-\mu \mathrm{m}$-thick sections and deparaffinized and stained using a fully automated Ventana BenchMark ULTRA stainer (Ven- tana, Tucson, Ariz., USA) according to the manufacturers' instructions at the Pathology Department. Binding of peroxidase-coupled antibodies was detected using 3,39-diaminobenzidine as a substrate, and the sections were counterstained with hematoxylin. The rabbit monoclonal anti-sst ${ }_{2}$ antibody (BioTrend, Köln, Germany) was used at a dilution of 1:25, whereas the rabbit monoclonal antisst $_{5}$ antibody at a dilution of 1:50.

Normal pancreatic tissue served as a positive control for both SSTR2 and SSTR5 staining. For negative controls, the primary antibody was omitted. Immunostaining of the somatotroph adenoma tissues was scored by a semiquantitative immunoreactivity scoring system (IRS) [18] and is the product of the percentage of positive-stained cells ( 0 : no positive cells; $1:<10 \% ; 2: 10-50 \%$; 3: $51-80 \% ; 4: 80 \%$ ) and the staining intensity (0: no staining; 1: weak staining; 2: moderate staining; 3: strong staining) (fig. 2).
Combination Treatment and SSTR

Expression in Acromegaly
Neuroendocrinology 2017;105:44-53 DOI: $10.1159 / 000448429$ 
Table 1. Patient characteristics - medical pretreatment at time of surgery

\begin{tabular}{|c|c|c|c|c|}
\hline & Naive & Mono-LA-SSA & LA-SSA + PEGV & $\mathrm{p}$ value \\
\hline Tissues & $23(59.0)$ & $9(23.1)$ & 7 (17.9) & \\
\hline \multicolumn{5}{|l|}{ Baseline characteristics } \\
\hline Males & $13(56.5)$ & $7(77.8)$ & $4(57.1)$ & 0.652 \\
\hline Tumor volume - macro & $22(95.7)$ & $9(100)$ & $6(85.7)$ & 0.379 \\
\hline Tumor volume - largest diameter ${ }^{\mathrm{a}}, \mathrm{mm}$ & $27.5(17.0-40.0)$ & $26.5(25.0-27.0)$ & $14.5(13.0-16.0)$ & 0.061 \\
\hline IGF-I xULN at diagnosis ${ }^{\mathrm{b}}$ & $2.8(2.7-3.1)$ & $3.8(2.7-5.5)$ & $3.1(2.8-4.5)$ & 0.247 \\
\hline \multicolumn{5}{|l|}{ Time of surgery } \\
\hline Age at time of surgery, years & $43.2(29.9-49.8)$ & $43.8(36.2-46.3)$ & $43.2(36.7-47.1)$ & 0.773 \\
\hline IGF-I xULN level before surgery & $2.7(2.5-3.2)$ & $2.6(1.7-3.8)$ & $1.9(1.3-2.4)$ & 0.098 \\
\hline Duration of LA-SSA before surgery ${ }^{c}$, months & n.a. & $6.0(4.0-9.0)$ & $20.0(18.5-24.0)$ & 0.094 \\
\hline \multicolumn{5}{|l|}{ Immunohistochemistry } \\
\hline SSTR2 IRS ${ }^{\mathrm{d}}$ & $6.0(2.0-12.0)$ & $6.0(6.0-12.0)$ & $2.0(1.0-4.5)$ & \\
\hline SSTR5 IRS & $12.0(7.0-12.0)$ & $12.0(6.0-12.0)$ & $9.0(9.0-12.0)$ & \\
\hline
\end{tabular}

Data are presented as n (\%) or median (IQR). Naive = No previous medical treatment; LA-SSA = long-acting somatostatin analogues; PEGV = pegvisomant; IGF-I = insulin-like growth factor I; ULN = upper limit of normal; n.a. = not applicable; IRS = immunoreactivity score; SSTR2 = somatostatin receptor subtype 2; SSTR5 = somatostatin receptor subtype $5 .{ }^{a}$ The largest diameter was missing in $56.5 \%$ of the patients in the naive group, $33.0 \%$ in the mono-LA-SSA group and $14.3 \%$ in the LA-SSA + PEGV group. ${ }^{\mathrm{b}}$ The IGF-I level expressed as ULN at baseline was missing in $21.7 \%$ of the patients in the naive group. In the other two groups all data were available. ${ }^{\mathrm{c}}$ The monoLA-SSA group includes 7 patients that used the maximum dose of LA-SSA and 2 patients which used Sandostatin LAR 20 mg monthly for 5 and 6 months. All patients in the combined treatment group used the maximum dose of LA-SSA. ${ }^{\mathrm{d}}$ Scoring system which takes into account both the percentage of positive cells and the intensity of the staining.

The IRS ranges between 0 and 12 . The somatotroph adenoma tissue scoring was performed by 2 independent investigators (S.E.F and F.G.), who were blinded to each other's findings, patient characteristics and their treatment regimes.

\section{Statistical Methods}

Data are expressed as median (IQR). Differences between two subgroups were analyzed using an unpaired t test or the MannWhitney $U$ test (in case of nonparametric data). Differences between three independent subgroups were analyzed using one-way ANOVA or the Kruskal-Wallis test (in case of nonparametric data). Nominal variables were analyzed using Fisher's exact test. Results of correlation analyses were expressed as Spearman's rank correlation coefficients (rho). p values $<0.05$ (two-tailed) were considered statistically significant. Statistical analyses were performed with SPSS version 20 (IBM SPSS Statistics for Windows, Armonk, N.Y., USA) and GraphPad Prism version 6 for Windows (GraphPad Software, San Diego, Calif., USA).

\section{Results}

\section{Patient Characteristics before Surgery}

Patient characteristics before surgery are presented in table 1 . Out of the 39 evaluated somatotroph adenoma tissue samples, 23 were collected during a drug-naive state, 9 during mono-LA-SSA therapy and 7 during LA-
SSAs combined with PEGV therapy. No significant differences were present between the three medical pretreatment groups before surgery when considering sex, tumor volume assessed as macro- versus microadenomas, IGF-I levels at diagnosis, age at time of surgery, IGF-I levels before surgery and the duration of LA-SSA treatment before surgery. IGF-I levels are expressed as xULN.

\section{Expression of SSTR2 and SSTR5 after Different} Medical Pretreatment Options

The SSTR2 IRS in the naive group had a median of 6.0 (2.0-12.0), the mono-LA-SSA group had a median of 6.0 (6.0-12.0), and the group pretreated with LA-SSA and PEGV had a median of 2.0 (1.0-4.5) (fig. 3a). A pairwise comparison showed that the median SSTR2 IRS on somatotroph adenomas was statistically significantly higher in the treatment-naive group compared to the combined group $(\mathrm{p}=0.048)$. We did not find any statistically significant difference in the SSTR2 IRS between the treatment-naive and mono-LA-SSA groups. However, a trend for a lower median SSTR2 IRS was observed in the combined group when compared to the mono-LA-SSA group $(\mathrm{p}=0.055)$.

\section{8}

Neuroendocrinology 2017;105:44-53 DOI: $10.1159 / 000448429$
Franck et al. 
Table 2. Patient characteristics - partial and full responders during monotherapy with LA-SSA

\begin{tabular}{lccc}
\hline & Partial LA-SSA responders & Full LA-SSA responders & p value \\
\hline Tissues & $31(79.5)$ & $8(20.5)$ & $5(62.5)$ \\
Males & $19(61.3)$ & $43.5(42.1-49.7)$ & 0.640 \\
Age at time of surgery, years & $43.2(30.7-49.9)$ & $8(87.5)$ & 0.306 \\
Tumor volume - macro & $30(96.8)$ & $7(87.5)$ & 0.372 \\
Medical pretreatment before surgery & $16(51.6)$ & $1(12.5)$ & 0.225 \\
$\quad$ Naive & $8(25.8)$ & 0 & 0.853 \\
$\quad$ Mono-LA-SSA & $7(22.8)$ & $0.7(0.5-3.2)$ & $\leq 0.001$ \\
LA-SSA + PEGV & $2.3(1.8-3.2)$ & \\
IGF-I xULN before surgery & $2.0(1.5-2.3)$ & & \\
IGF-I xULN during mono-LA-SSA & & & \\
\hline
\end{tabular}

Data are presented as n (\%) or median (IQR). Partial responders to LA-SSA were considered to have elevated serum IGF-I levels after at least 6 months on the highest dose of LA-SSAs and needed PEGV for disease control. Full responders to LA-SSA achieved normal IGF-I levels during monotherapy of LA-SSA and PEGV was added because of quality of life reasons during a clinical trial. Monotherapy of LA-SSA could have been administered to the patient before or after surgery. Naive = No previous medical treatment; LA-SSA = longacting somatostatin analogues; PEGV = pegvisomant; , IGF-I = insulin-like growth factor I; ULN = upper limit of normal. ${ }^{a}$ In order to subdivide the total cohort into partial and full responders, the response of IGF-I levels to monotherapy with LA-SSA could have been evaluated before or after surgery.

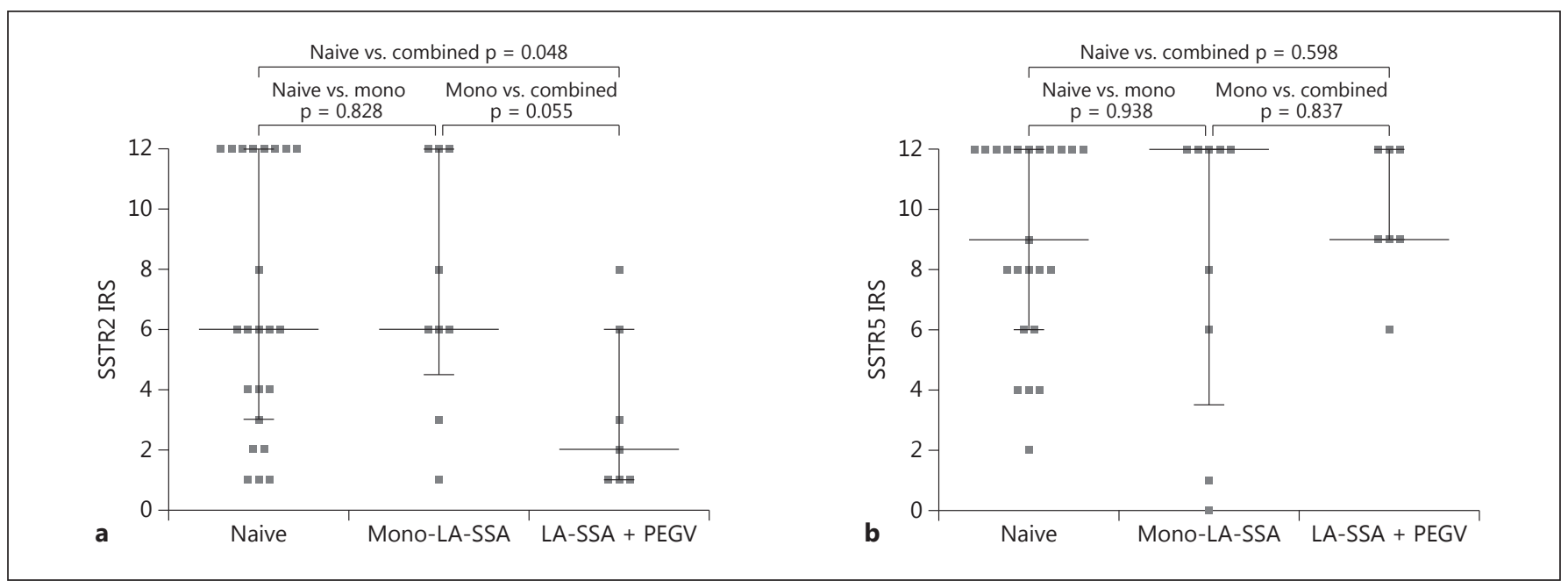

Fig. 3. SSTR2 and SSTR5 expression per medical pretreatment group. a The SSTR2 IRS was significantly lower in the combination group compared to the naive group. $\mathbf{b}$ The SSTR5 IRS was not significantly different between the different medical pretreatment groups. SSTR IRS is expressed as median (interquartile range). Pretreatment groups before surgery were tested reciprocally with

The SSTR5 IRS in the naive group had a median of 12.0 (7.0-12.0), the mono-LA-SSA group had a median of 12.0 (6.0-12.0), and the group pretreated with LA-SSA and PEGV had a median of $9.0(9.0-12.0)$. The SSTR5 IRS did not significantly differ between LA-SSA + PEGV treatment and the other two groups (fig. $3 \mathrm{~b}$ ).

Combination Treatment and SSTR

Expression in Acromegaly the Mann-Whitney $\mathrm{U}$ test (naive: $\mathrm{n}=23$; mono-LA-SSA: $\mathrm{n}=9$; LA-SSA + PEGV: $n=7$ ). Naive $=$ No previous medical treatment; LA-SSA = long-acting somatostatin analogues; PEGV = pegvisomant; IGF-I = insulin-like growth factor I; ULN = upper limit of normal; SSTR2 = somatostatin receptor subtype 2; SSTR5 = somatostatin receptor subtype 5 ; IRS = immunoreactivity score.

\section{Partial and Full Responders to Monotherapy with LA-SSAs}

All patients selected for this study were eventually treated with LA-SSAs combined with PEGV after surgery and were subdivided into partial and full responders to monotherapy with LA-SSAs. Partial-responder 


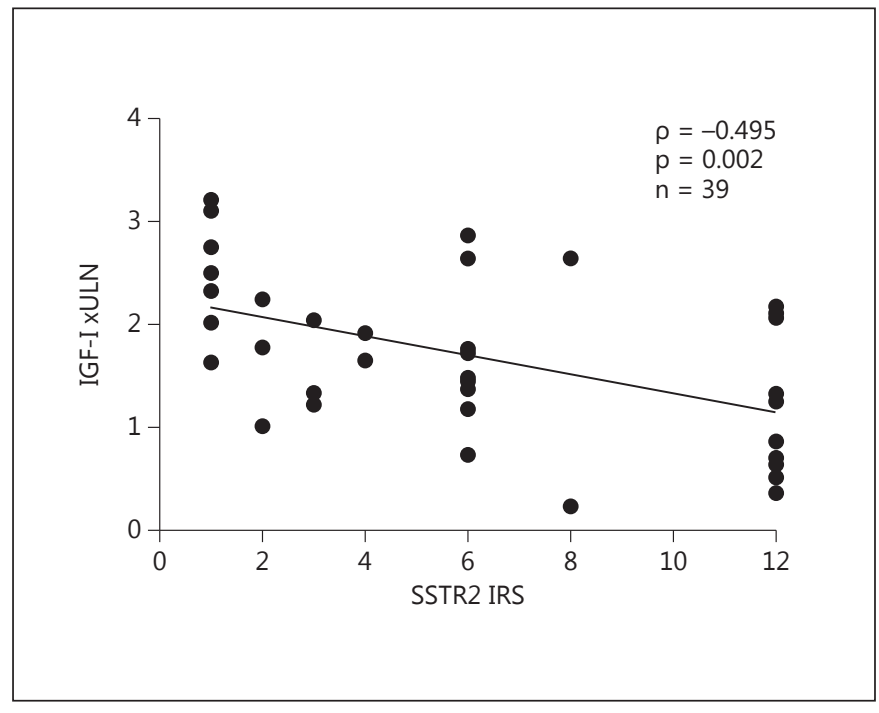

Fig. 4. IGF-I xULN during the highest dose of monotherapy with LA-SSA was inversely correlated with the SSTR2 expression (Spearman correlation analyses). LA-SSA = Long-acting somatostatin analogues; IGF-I = insulin-like growth factor I; ULN = upper limit of normal; SSTR2 = somatostatin receptor subtype 2; IRS = immunoreactivity score.

patients had elevated serum IGF-I levels after at least 6 months on LA-SSAs and needed PEGV in combination with LA-SSAs in order to achieve normalized IGF-I levels. Full responders to LA-SSA achieved normal IGF-I levels during monotherapy with LA-SSA. Patient characteristics of partial $(n=31)$ and full responders $(n=8)$ to monotherapy with LA-SSA are presented in table 2. No major differences were observed between partial and full responders to monotherapy with LA-SSAs regarding sex, age at time of surgery, tumor volume assessed as macro versus microadenomas, medical pretreatment before surgery and IGF-I xULN at time of surgery. IGFI xULN during monotherapy with LA-SSA was significantly different, as we selected this variable. As for the SSTR expression at the time of surgery, when only partial-responder patients were included, we observed that the SSTR2 and SSTR5 IRS followed a similar distribution between the medical pretreatment groups to the one shown in the previous paragraph. However, possibly due to the loss of statistical power (8 patients less were included in the analysis), the difference in the SSTR2 IRS between the naive and the combined group was not significant anymore $(\mathrm{p}=0.135)$ (see online suppl. fig. 1; for all online suppl. material see www. karger.com/doi/10.1159/000448429).

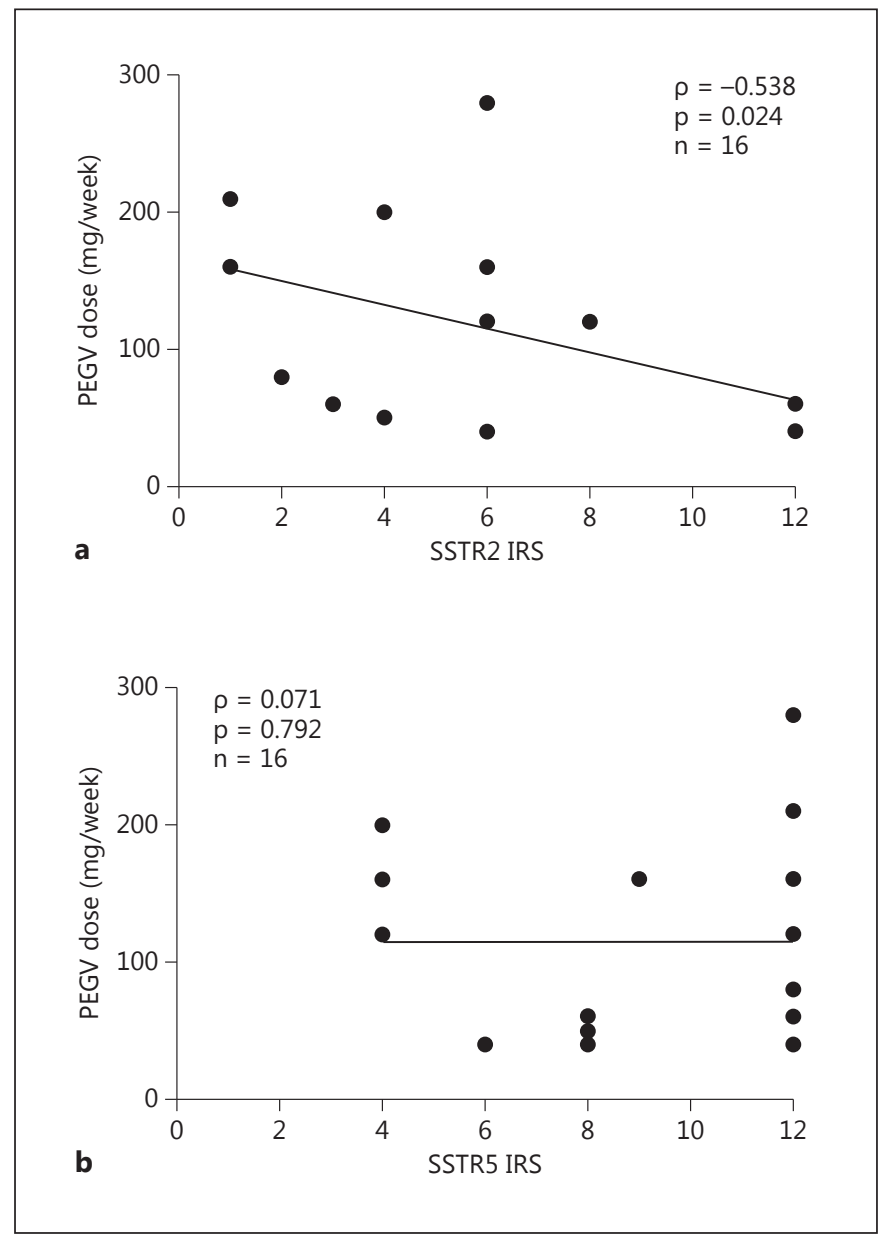

Fig. 5. a The required PEGV dose (mg/week) in combination with the highest dose of LA-SSA needed to control IGF-I levels was inversely correlated with the expression of SSTR2 (Spearman correlation analyses). This figure only contains partial responders to monotherapy with LA-SSA, and the SSTR expression was estimated during a drug-naive state $(n=16)$. $\mathbf{b}$ The expression of SSTR5 was not correlated with the required PEGV dose. PEGV = Pegvisomant; LA-SSA = long-acting somatostatin analogues; SSTR2 = somatostatin receptor subtype 2 ; IRS = immunoreactivity score.

\section{IGF-I Levels and PEGV Dosing in Relation to the SSTR 2 and SSTR5 Expression}

IGF-I levels (xULN) during monotherapy with the highest approved dose of LA-SSAs were inversely correlated with the SSTR2 expression $(\rho=-0.495, p=0.002$, $\mathrm{n}=39$; fig. 4). The SSTR5 expression was not correlated with the IGF-I (xULN) during monotherapy with LASSA $(\rho=0.145, p=0.405, n=39)$. As mentioned in the Methods section, when observing the required PEGV dose to achieve IGF-I normalization in relation to the SSTR2 and SSTR5 expression, we included only the par-
Franck et al. 
tial-responder patients, not pretreated before surgery (drug-naive status). In this context, we observed that the required PEGV dose was inversely correlated with the SSTR2 expression ( $\rho=-0.538, p=0.024, n=16$; fig. $5 a)$, while it did not correlate with the SSTR5 expression ( $\rho=$ $-0.071, p=0.792, n=16$; fig. $5 b$ ). The correlation analyses of the medical pretreatment groups before surgery [monotherapy with LA-SSA $(\mathrm{n}=9)$ and LA-SSA in combination with PEGV $(n=7)$ ], as described in the first part of this results section, did not reveal statistical significance between the required PEGV dose and the SSTR2 and SSTR5 expression.

\section{Discussion}

This is the first study aimed to investigate the expression of SSTR2 and SSTR5 in somatotroph adenoma tissues of acromegalic patients treated with LA-SSAs in combination with PEGV. In our specific cohort, the combined group (LA-SSA/PEGV) showed a lower median SSTR2 IRS at the time of surgery compared to the drugnaive group, while the IRS of the SSTR 5 was not different between the pretreatment groups. Additionally, we observed that the required PEGV dose to achieve normalization of IGF-I levels was inversely correlated with the SSTR2 expression, but not with the IRS of SSTR5 in drugnaive acromegalic patients. In the following discussion, we focus on all the possible explanations for the finding that in our cohort the SSTR2 expression was lower in the combined group compared with the naive group. The most likely explanation for this finding is that somatotroph adenomas (partially) resistant to LA-SSA, which need combined treatment, have lower SSTR2 expression at baseline. However, we cannot exclude that other factors, such as feedback mechanisms of the GH-IGF-I axis and the downregulation of SSTR2 due to prior LA-SSA treatment could affect the SSTR expression as well.

As far as the GH-IGF-I axis feedback mechanisms play a possible role in the modulation of SSTR expression, we assume that $\mathrm{GH}$ itself does not have a direct effect on the modulation of SSTRs at the pituitary level during combination treatment, since PEGV blocks GH receptors also at the level of the pituitary [19]. More complex is the prediction of GHRH levels via the hypothalamus in the presence of PEGV treatment. PEGV generally causes a further elevation of serum GH levels [20], which could result in a decrease in GHRH via some areas in the hypothalamus and might consequently result in a downregulation of SSTR2. On the other hand, the drop of IGF-I and the as-

Combination Treatment and SSTR

Expression in Acromegaly sociated increase in hypothalamic GHRH levels might have an influence on the expression of SSTR2 and SSTR5 at the level of the somatotroph adenoma as well. In our study, IGF-I levels of patients treated with LA-SSA in combination with PEGV were relatively lower compared to the naive group. However, Park et al. [21] reported that in spontaneous dwarf rats, the expression of all SSTR subtypes was not directly influenced by exogenous IGF-I treatment. Moreover, in the same study, the authors observed that GHRH has a direct stimulatory effect on SSTR2 expression, both in vivo and in vitro. If these observations can be extrapolated to humans, we could speculate that the decrease in IGF-I levels probably does not play a major role in the explanation of the observed decrease in SSTR2 expression during the combination treatment, while the modulation of GHRH levels seems to be more involved. However, based on the current knowledge, our results demonstrating a lower SSTR2 expression in the combined group compared to the naive group cannot clearly be explained by the effect of PEGV on the modulation of the GH-IGF-I-pituitary-hypothalamus axis. Finally, to the best of our knowledge, a direct effect of PEGV on SSTR expression has not been reported, although it cannot be excluded a priori.

The downregulation of cell surface SSTR2 by endogenous somatostatin and LA-SSA treatment has been reported in several studies $[7,22,23]$, possibly through ligand-induced receptor internalization. Casar-Borota et al. [7] demonstrated this downregulation of SSTR2 expression by LA-SSA therapy also in a randomized subset of acromegalic patients ( $\mathrm{n}=13$ mono-LA-SSA, $\mathrm{n}=13$ direct surgery), to exclude a possible clinical selection bias. However, we did not observe a statistically significant difference in SSTR2 expression between the naive and monoLA-SSA pretreatment groups in our acromegaly cohort $(\mathrm{p}=0.828)$. A possible explanation for this finding may reside in the selection of our patient group. Indeed, our cohort is most likely represented by acromegalic patients with more disease activity, since they were all referred to us as a tertiary referral hospital and, most importantly, the majority of these patients needed PEGV in addition to LA-SSAs to normalize their IGF-I levels $(\mathrm{n}=31)$. In this light, as shown in the result section, even the drug-naive group showed a relatively low SST2 IRS (median IRS 6, which means about $50 \%$ of moderately stained cells) compared to the staining observed in a previous study using comparable techniques and scoring system (median IRS 9) [7]. This finding may result in a lower downregulation of SSTR2 after LA-SSA treatment alone and/or contribute to 'mask' the downregulation of a receptor pattern already

Neuroendocrinology 2017;105:44-53 DOI: $10.1159 / 000448429$ 
relatively low at baseline. However, considering all these limitations, we observed that the combined treatment group in our study had a lower SSTR2 IRS compared to the naive group. Noteworthy, the SSTR2 IRS was almost significantly different $(p=0.055)$ between the mono-LASSA and combined groups. Furthermore, the duration of LA-SSA treatment was remarkably shorter in the monoLA-SSA group compared to the combined treatment group by a median difference of 14 months; however, this difference did not reach statistical significance. This difference could be a possible explanation (besides the concomitant treatment with PEGV) for the observed lower expression of SSTR2 in the combined treatment group compared to the mono-LA-SSA and naive groups. Thus, the lower expression of SSTR2 in the somatotroph adenomas in the combined treatment group could simply be related to the fact that these patients necessitate combined treatment due to their (partial) resistance to LA-SSA treatment. Therefore, we can hypothesize that these partial responders to monotherapy with LA-SSA already have a lower SSTR2 expression at baseline, which could be the reason why these patients need PEGV in addition to LASSA for disease control. It has already been reported that full responders to LA-SSA have a significantly higher SSTR2 expression compared to partial LA-SSA responders $[9,12]$. Furthermore, for the first time, we observed that in drug-naive somatotroph adenomas, a lower SSTR2 expression correlates with a higher required PEGV dose in order to achieve normalized IGF-I levels, which also reflects more severe disease activity. This observation may have clinical implications for the postoperative treatment of acromegalic patients and, therefore, represents an important finding of our study.

The main limitations of this study are (1) the retrospective design, (2) the relative small sample size and (3) the peculiar patient group in which the study has been conducted (all treated with combination medical therapy during their clinical history). However, this can be expected due to the rarity of acromegaly as well as the fact that only a subset of the patients is treated with LA-SSA in combination with PEGV. These limitations of the present study could be overcome in the future by the design of a large prospective randomized study aimed to evaluate the impact of different treatment modalities on SSTR expression. Furthermore, the assessment of IGF-I and $\mathrm{GH}$ levels can also be improved, and, in particular, GH measurement should be assessed by a noncommercial assay in order to distinguish between endogenous $\mathrm{GH}$ and PEGV, which has recently been introduced in our clinical practice.

\section{Conclusion}

This is the first study that assessed the effect of the combined medical treatment with LA-SSAs and PEGV on SSTR expression in somatotroph adenoma tissues of acromegalic patients. In our specific cohort, the SSTR2 expression was lower in patients pretreated with LA-SSA/ PEGV compared to the drug-naive acromegalic patients. This finding is in line with the evidence that LA-SSA/ PEGV-treated patients are usually (partially) resistant to LA-SSA treatment alone (depending on SSTR2 expression). Moreover, we observed that patients with a lower SSTR2 expression need a higher required PEGV dose in combination with LA-SSA to achieve normalized IGF-I levels after surgery, when assessed in drug-naive somatotroph adenoma tissues.

\section{Acknowledgements}

The authors would like to thank all patients for their participation. They would also like to thank B. Poldermans from the Department of Endocrinology for her support during blood collection, L. de Vogel from the Department of Pathology, and Dr. E.J. Delwel and Dr. J.H. van den Berge from the Department of Neurosurgery for their support in collecting somatotroph adenoma tissues.

\section{Disclosure Statement}

F. Gatto has received speaker's fees from Novartis. A.J. van der Lely and S.J.C.M.M. Neggers have received financial support for investigator-initiated research, unrestricted grants and speaker's fees from Novartis, Pfizer and Ipsen. A.J. van der Lely also received consultancy fees from Novartis and Pfizer. L.J. Hofland has received investigator-initiated research grants from Novartis and Ipsen. S.E. Franck, A.H.G. Dallenga, A.P. Nagtegaal and J.A.M.J.L. Janssen have nothing to disclose.

References

1 Schaer JC, Waser B, Mengod G, Reubi JC: Somatostatin receptor subtypes sst1, sst2, sst3 and sst 5 expression in human pituitary, gastroentero-pancreatic and mammary tumors: Comparison of mRNA analysis with receptor autoradiography. Int J Cancer 1997;70:530537.

2 Giustina A, Chanson P, Kleinberg D, Bronstein MD, Clemmons DR, Klibanski A, van der Lely AJ, Strasburger CJ, Lamberts SW, Ho KK, Casanueva FF, Melmed S; Acromegaly Consensus Group: Expert consensus document: a consensus on the medical treatment of acromegaly. Nat Rev Endocrinol 2014;10: 243-248.

Franck et al. 
3 Katznelson L, Laws ER Jr, Melmed S, Molitch ME, Murad MH, Utz A, Wass JA, Endocrine Society: Acromegaly: an endocrine society clinical practice guideline. J Clin Endocrinol Metab 2014;99:3933-3951.

4 Carmichael JD, Bonert VS, Nuno M, Ly D, Melmed S: Acromegaly clinical trial methodology impact on reported biochemical efficacy rates of somatostatin receptor ligand treatments: a meta-analysis. J Clin Endocrinol Metab 2014;99:1825-1833.

5 Annamalai AK, Webb A, Kandasamy N, Elkhawad M, et al: A comprehensive study of clinical, biochemical, radiological, vascular, cardiac, and sleep parameters in an unselected cohort of patients with acromegaly undergoing presurgical somatostatin receptor ligand therapy. J Clin Endocrinol Metab 2013;98: 1040-1050.

6 Caron PJ, Bevan JS, Petersenn S, Flanagan D, Tabarin A, Prevost G, Maisonobe P, Clermont A: Tumor shrinkage with lanreotide autogel $120 \mathrm{mg}$ as primary therapy in acromegaly: results of a prospective multicenter clinical trial. J Clin Endocrinol Metab 2014;99: 1282-1290.

7 Casar-Borota O, Heck A, Schulz S, Nesland JM, Ramm-Pettersen J, Lekva T, Alafuzoff I, Bollerslev J: Expression of SSTR2A, but not of SSTRS 1,3 , or 5 in somatotroph adenomas assessed by monoclonal antibodies was reduced by octreotide and correlated with the acute and long-term effects of octreotide. J Clin Endocrinol Metab 2013;98:E1730-E1739.

8 Ferone D, de Herder WW, Pivonello R, Kros JM, van Koetsveld PM, de Jong T, Minuto F, Colao A, Lamberts SW, Hofland LJ: Correlation of in vitro and in vivo somatotropic adenoma responsiveness to somatostatin analogs and dopamine agonists with immunohistochemical evaluation of somatostatin and dopamine receptors and electron microscopy. J Clin Endocrinol Metab 2008;93:14121417.
9 Gatto F, Feelders RA, van der Pas R, Kros JM, Waaijers M, Sprij-Mooij D, Neggers SJ, van der Lelij AJ, Minuto F, Lamberts SW, de Herder WW, Ferone D, Hofland LJ: Immunoreactivity score using an anti-sst $2 \mathrm{~A}$ receptor monoclonal antibody strongly predicts the biochemical response to adjuvant treatment with somatostatin analogs in acromegaly. J Clin Endocrinol Metab 2013;98:E66E71.

10 Reubi JC, Landolt AM: The growth hormone responses to octreotide in acromegaly correlate with adenoma somatostatin receptor status. J Clin Endocrinol Metab 1989;68:844850.

11 Taboada GF, Luque RM, Neto LV, Machado Ede O, Sbaffi BC, Domingues RC, Marcondes JB, Chimelli LM, Fontes R, Niemeyer P, de Carvalho DP, Kineman RD, Gadelha MR: Quantitative analysis of somatostatin receptor subtypes (1-5) gene expression levels in somatotropinomas and correlation to in vivo hormonal and tumor volume responses to treatment with octreotide LAR. Eur J Endocrinol 2008;158:295-303.

12 Casarini AP, Jallad RS, Pinto EM, Soares IC, Nonogaki S, Giannella-Neto D, Musolino NR, Alves VA, Bronstein MD: Acromegaly: correlation between expression of somatostatin receptor subtypes and response to octreotide-lar treatment. Pituitary 2009;12:297303.

13 Saveanu A, Gunz G, Dufour H, Caron P, Fina F, Ouafik L, Culler MD, Moreau JP, Enjalbert A, Jaquet P: Bim-23244, a somatostatin receptor subtype 2- and 5-selective analog with enhanced efficacy in suppressing growth hormone (GH) from octreotide-resistant human $\mathrm{GH}$-secreting adenomas. J Clin Endocrinol Metab 2001;86:140-145.

14 Neggers SJ, Franck SE, de Rooij FW, Dallenga AH, Poublon RM, Feelders RA, Janssen JA, Buchfelder M, Hofland LJ, Jorgensen JO, van der Lely AJ: Long-term efficacy and safety of pegvisomant in combination with long-acting somatostatin analogs in acromegaly. J Clin Endocrinol Metab 2014;99:3644-3652.

15 van der Lely AJ, Hutson RK, Trainer PJ, Besser GM, et al: Long-term treatment of acromegaly with pegvisomant, a growth hormone receptor antagonist. Lancet 2001;358:17541759.
16 Neggers SJ, van Aken MO, de Herder WW, Feelders RA, Janssen JA, Badia X, Webb SM, van der Lely AJ: Quality of life in acromegalic patients during long-term somatostatin analog treatment with and without pegvisomant. J Clin Endocrinol Metab 2008;93:3853-3859.

17 Elmlinger MW, Kuhnel W, Weber MM, Ranke MB: Reference ranges for two automated chemiluminescent assays for serum insulinlike growth factor I (IGF-I) and IGF-binding protein 3 (IGFBP-3). Clin Chem Lab Med 2004;42:654-664.

18 Remmele W, Stegner HE: Recommendation for uniform definition of an immunoreactive score (IRS) for immunohistochemical estrogen receptor detection (ER-ICA) in breast cancer tissue (in German). Pathologe 1987;8:138-140.

19 Melmed S: The Pituitary, ed 2. Oxford, Blackwell Science, 2002, chapter 1, p 4.

20 Madsen M, Fisker S, Feldt-Rasmussen U, Andreassen M, Kristensen LO, Orskov H, Jorgensen JO: Circulating levels of pegvisomant and endogenous growth hormone during prolonged pegvisomant therapy in patients with acromegaly. Clin Endocrinol 2014;80:92-100.

21 Park S, Kamegai J, Johnson TA, Frohman LA, Kineman RD: Modulation of pituitary somatostatin receptor subtype (sst1-5) messenger ribonucleic acid levels by changes in the growth hormone axis. Endocrinology 2000; 141:3556-3563.

22 Dournaud P, Boudin H, Schonbrunn A, Tannenbaum GS, Beaudet A: Interrelationships between somatostatin sst $2 \mathrm{~A}$ receptors and somatostatin-containing axons in rat brain: evidence for regulation of cell surface receptors by endogenous somatostatin. J Neurosci 1998;18:1056-1071.

23 Fougner SL, Borota OC, Berg JP, Hald JK, Ramm-Pettersen J, Bollerslev J: The clinical response to somatostatin analogues in acromegaly correlates to the somatostatin receptor subtype 2a protein expression of the adenoma. Clin Endocrinol 2008;68:458-465.
Combination Treatment and SSTR

Expression in Acromegaly
Neuroendocrinology 2017;105:44-53

DOI: $10.1159 / 000448429$ 